Mon. Not. R. Astron. Soc. 000, 1, 8 (2011) Printed 11 October $2018 \quad$ (MN LTEX style file v2.2)

\title{
Swift/XRT monitoring of the candidate Supergiant Fast X-ray Transient IGR J16418-4532
}

\author{
P. Romano ${ }^{1}$, V. Mangano ${ }^{1}$, L. Ducci ${ }^{2}$, P. Esposito ${ }^{3}$, P.A. Evans ${ }^{4}$, S. Vercellone ${ }^{1}$, \\ J.A. Kennea ${ }^{5}$, D.N. Burrows ${ }^{5}$, N. Gehrels ${ }^{6}$ \\ ${ }^{1}$ INAF, Istituto di Astrofisica Spaziale e Fisica Cosmica, Via U. La Malfa 153, I-90146 Palermo, Italy \\ ${ }^{2}$ Institut für Astronomie und Astrophysik, Universität Tübingen, Sand 1, D-72076 Tübingen, Germany \\ ${ }^{3}$ INAF, Osservatorio Astronomico di Cagliari, località Poggio dei Pini, Strada 54, I-09012 Capoterra, Italy \\ ${ }^{4}$ Department of Physics \& Astronomy, University of Leicester, LE1 7RH, UK \\ ${ }^{5}$ Department of Astronomy and Astrophysics, Pennsylvania State University, University Park, PA 16802, USA \\ ${ }^{6}$ NASA/Goddard Space Flight Center, Greenbelt, MD 20771, USA
}

Accepted 2011 September 28. Received 2011 September 28; in original form 2011 August 17

\begin{abstract}
We report on the Swift monitoring of the candidate supergiant fast X-ray transient (SFXT) IGR J16418-4532, for which both orbital and spin periods are known $(\sim 3.7 \mathrm{~d}$ and $\sim 1250 \mathrm{~s}$, respectively). Our observations, for a total of $\sim 43 \mathrm{ks}$, span over three orbital periods and represent the most intense and complete sampling of the light curve of this source with a sensitive X-ray instrument. With this unique set of observations we can address the nature of this transient. By applying the clumpy wind model for blue supergiants to the observed $\mathrm{X}$-ray light curve, and assuming a circular orbit, the X-ray emission from this source can be explained in terms of the accretion from a spherically symmetric clumpy wind, composed of clumps with different masses, ranging from $\sim 5 \times 10^{16} \mathrm{~g}$ to $10^{21} \mathrm{~g}$. Our data suggest, based on the X-ray behaviour, that this is an intermediate SFXT.
\end{abstract}

Key words: X-rays: binaries - X-rays: individual (IGR J16418-4532)

\section{INTRODUCTION}

The X-ray transient IGR J16418-4532 was discovered by INTEGRAL during observations of the black hole X-ray transient 4U 1630-47 on 2003 February 1-5 (Tomsick et al. 2004), with a 20-40 keV flux of $3 \times 10^{-11} \mathrm{erg} \mathrm{cm}^{-2} \mathrm{~s}^{-1}$. Sguera et al. (2006) reported on further INTEGRAL observations, taken on 2004 February 26, during which fast $(\sim 1 \mathrm{hr}) \mathrm{X}$-ray outbursts were observed that peaked at $\sim 80 \mathrm{mCrab}(20-30 \mathrm{keV})$. This behavior led Sguera et al. (2006) to propose that IGR J16418-4532 is a candidate supergiant fast X-ray transient (SFXT). SFXTs (e.g. Sguera et al. 2005) are high mass X-ray binaries (HMXBs) associated with OB supergiant stars via optical spectroscopy that show an X-ray dynamic range of 3-5 orders of magnitude. Indeed, they are characterized by bright (peak luminosities of $10^{36}-10^{37} \mathrm{erg} \mathrm{s}^{-1}$ ), short (a few hours, as observed by INTEGRAL, Sguera et al. 2005; Negueruela et al. 2006) $\mathrm{X}$-ray outbursts significantly shorter than those of typical $\mathrm{Be} / \mathrm{X}$ ray binaries, and a quiescent luminosity of $\sim 10^{32} \mathrm{erg} \mathrm{s}^{-1}$ (e.g. in't Zand 2005; Bozzo et al. 2010).

In their systematic analysis of the INTEGRAL observations from 2003 to 2009, Ducci et al. (2010) detected 23 outbursts, for an activity duty cycle of $\sim 1 \%$ (one of the highest among the 14 SFXTs and SFXT candidates they examined) and fluxes ranging between $1.3 \times 10^{-10} \mathrm{erg} \mathrm{cm}^{-2} \mathrm{~s}^{-1}$ and $4.8 \times 10^{-10} \mathrm{erg} \mathrm{cm}^{-2}$ $\mathrm{s}^{-1}(18-100 \mathrm{keV})$.

XMM-Newton observations (Walter et al. 2006) showed a heavily absorbed $\left(N_{\mathrm{H}}=(1.0 \pm 0.1) \times 10^{23} \mathrm{~cm}^{-2}\right)$ pulsar $\left(P_{\text {spin }}=\right.$ $1246 \pm 100 \mathrm{~s}$ ) with an unabsorbed flux of $1.3 \times 10^{-10} \mathrm{erg} \mathrm{cm}^{-2} \mathrm{~s}^{-1}$ $(2-100 \mathrm{keV})$ in a fit performed in conjunction with the INTEGRAL data.

Chaty et al. (2008) proposed, based on the XMM-Newton position, 2MASS J16415078-4532253 as the best NIR counterpart candidate; they also fitted the spectral energy distribution (SED), obtaining a temperature of $32800 \mathrm{~K}$ for the massive companion, consistent with an OB spectral classification. SED fitting by Rahoui et al. (2008) yielded $A_{V}=14.5 \mathrm{mag}$, and a O8.5 spectral type; depending on the luminosity class they derive a distance of 4.9 (V), 8.3 (III), or $13 \mathrm{kpc}$ (I).

A periodicity of $\sim 3.75 \mathrm{~d}$ was discovered by Corbet et al. (2006) in the Swift/Burst Alert Telescope (BAT, Barthelmy et al. 2005) (3.753 $\pm 0.004 \mathrm{~d}$, MJD 53360-53630) and RXTE/ASM (3.7389 $\pm 0.0004 \mathrm{~d}$, MJD 50091-53810) light curves, which was interpreted as the orbital period of a binary system. The hint of a total eclipse at phase 0.5 observed in the BAT data is consistent with a supergiant companion. Levine et al. (2010) refined the orbital period value to $3.73886 \pm 0.00028 \mathrm{~d}$, by using the $R X T E / A S M$ data up to MJD 55224. The observed values of the orbital and pulse periods 
Table 1. Summary of the Swift/XRT observations.

ObsID Start time (UT) End time (UT) $\quad$ Exp. $\phi^{a}$

(s)

\begin{tabular}{|c|c|c|c|c|}
\hline & & & & \\
\hline & & & & \\
\hline & & & & \\
\hline & & & & \\
\hline & & & 4832 & \\
\hline & & & 273 & \\
\hline & & & 1078 & 72 \\
\hline $06^{d}$ & & & & \\
\hline & & & 37 & 01 \\
\hline & & & 1947 & 62 \\
\hline & & & 2673 & 77 \\
\hline & & & 34 & 0.0 \\
\hline & & & 3761 & 0.3 \\
\hline & & & 2812 & .6 \\
\hline & & & 4127 & 0.8 \\
\hline & 2011-07-29 12:13:30 & 2011-07-29 23:34:57 & 4650 & 0.5 \\
\hline 032037007 & 2011-07-30 01:01:30 & 2011-07-30 09:27:56 & 4287 & 0.6 \\
\hline
\end{tabular}

${ }^{\text {a }}$ Mean phase referred to $T_{\text {epoch }}=$ MJD 53560.2 (Levine et al. 2010).

b Corresponding to MJD 55610.08.

${ }^{\mathrm{c}}$ Serendipitous observation.

${ }^{\mathrm{d}}$ WT mode data.

indeed place IGR J16418-4532 in a region of the Corbet diagram (Corbet 1986) where wind-accreting HMXBs generally lie.

In this paper we analyze all the Swift data collected on IGR J16418-4532. The first set of observations comprises two $2 \mathrm{ks}$ target of opportunity (ToO) observations performed on 2011 February 18 and March 4, following a MAXI alert of a possible detection of IGR J16418-4532 on 2011 February 17 (preliminary results were described in Romano et al. 2011b). The second set is drawn from a monitoring campaign in 2011 July that spans over three orbital periods. This Swift monitoring represents the most intense and complete sampling along the orbital period of the light curve of this source with a sensitive X-ray instrument.

\section{OBSERVATIONS AND DATA REDUCTION}

Table 1 reports the $\log$ of the Swift/X-ray Telescope (XRT, Burrows et al. 2005) observations used in this paper. The Swift observations of IGR J16418-4532 during 2011 February and March (00031929001 and 00031929002) were obtained (Romano et al. 2011b) as two ToO observations following a MAXI alert (5609524433, later retracted, as due to GX $340+0)$ of a possible detection of IGR J16418-4532 on 2011 February 17. The data collected in 2011 July were obtained as a ToO monitoring program of 9 scheduled daily observations, each $5 \mathrm{ks}$ long, starting on 2011 July 13. This strategy was devised in order to cover the light curve in different orbital phases while maintaining the observing time per day reasonably short, thus not hindering gamma-ray burst (GRB) observations. However, most observations were cut short by the occurrence of GRBs and the discovery of a new soft gamma-ray repeater (starting from obsID 00031929005, see Table 1). Observation 00032037001 was performed to determine the best offset from the intended target in order to alleviate the problem of singlereflection rings from the nearby source GX $340+0$ (these rings marginally affect the source while in the faint states, so that the analysis of the earlier segments was still feasible, with an appropriate choice of extraction regions) A new pointing (hence a new obsID) was adopted afterwards. In our work we also include observation 00043105001 that serendipitously covers the region of IGR J16418-4532. The 2011 July campaign lasted 18 days divided in 12 observations for a total on-source exposure of $\sim 39$ ks.

For our binary orbital period search and analysis we retrieved the BAT 'orbit-by-orbit' light curves (averaged over Swift's orbital period of $\sim 90 \mathrm{~m}, 15-50 \mathrm{keV}$ ) covering the data range from 2005 February 12 to 2011 July 12 (MJD range 53413-55754) from the BAT Transient Monitor (Krimm et al. 2006, 2008) pag£. These data were further screened to exclude bad quality points (quality flag 1 and 2) and referred to the solar system barycentre (SSB) by using the task EARTH2SUN.

The XRT data were processed with standard procedures (XRTPIPELINE v0.12.6), filtering and screening criteria by using FTOOLS. Both WT and PC events were considered. The selection of event grades was $0-2$ and $0-12$, for WT and PC data, respectively (Burrows et al. 2005). Source events were accumulated within a annular/circular region (depending on whether pile-up correction was required or not, respectively) with an outer radius of 20 pixels ( 1 pixel $\left.\sim 2.36^{\prime \prime}\right)$; and background events were accumulated from source-free regions far from the contamination of the singlereflection rings from GX $340.0+0$. For our timing analysis we converted the event arrival times to the Solar system barycentre with the task BARYCORR. Light curves were created for several values of signal-to-noise ratio (SNR) and number of counts per bin; all were corrected for PSF losses, vignetting and background. For our spectral analysis, we extracted events in the same regions as those adopted for the light curve creation; ancillary response files were generated with XRTMKARF, to account for different extraction regions, vignetting, and PSF corrections. We used the latest spectral redistribution matrices in CALDB (20110705).

The UV/Optical Telescope (UVOT, Roming et al. 2005) observed IGR J16418-4532 simultaneously with the XRT with the 'Filter of the Day' (FoD), i.e. the filter chosen for all observations to be carried out during a specific day in order to minimize the filter wheel usage. The exception is observation 00032037007, which was requested in the $u$ filter, as opposed to FoD, to attempt to obtain a detection in that band. The data analysis was performed using the uvotimsum and UVOTSOURCE tasks included in the FTOOLS. The latter task calculates the magnitude through aperture photometry within a circular region and applies specific corrections due to the detector characteristics.

All quoted uncertainties are given at $90 \%$ confidence level for one interesting parameter unless otherwise stated. The spectral indices are parameterized as $F_{\nu} \propto \nu^{-\alpha}$, where $F_{\nu}\left(\mathrm{erg} \mathrm{cm}^{-2} \mathrm{~s}^{-1}\right.$ $\mathrm{Hz}^{-1}$ ) is the flux density as a function of frequency $\nu$; we adopt $\Gamma=\alpha+1$ as the photon index, $N(E) \propto E^{-\Gamma}\left(\mathrm{ph} \mathrm{cm}^{-2} \mathrm{~s}^{-1}\right.$ $\left.\mathrm{keV}^{-1}\right)$.

\section{RESULTS}

\subsection{Orbital period from BAT data}

For the orbital modulation, we considered the BAT data in the time range MJD 53414-55754. With a standard folding analy-

\footnotetext{
1 http://swift.gsfc.nasa.gov/docs/swift/results/transients/
} 


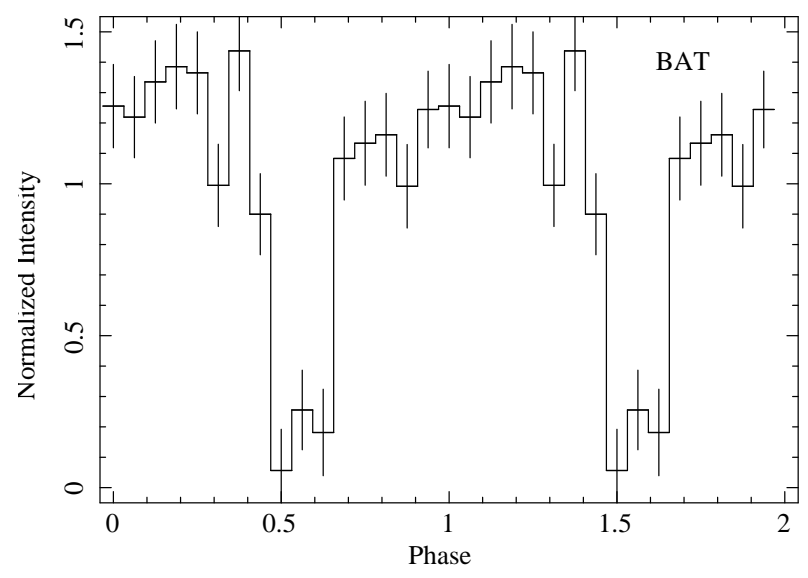

Figure 1. BAT light curve folded at $P_{\text {orb }}=3.73886 \mathrm{~d}$ and $T_{\text {epoch }}=$ MJD 53560.20000.

sis of the Solar system barycentred light curves, we measured an orbital period of $3.740 \pm 0.002$ days ( $1-\sigma$ error), which is fully consistent with the value obtained by Levine et al. (2010), $P_{\text {orb }}=3.73886 \pm 0.00028 \mathrm{~d}$. Therefore, hereafter we shall adopt the more precise measure by Levine et al. (2010), and we shall refer to $T_{\text {epoch }}=$ MJD 53560.20000 (Corbet et al. 2006). Fig. 1 shows the BAT light curve folded over this period.

The BAT data show an eclipse centred at $\phi \sim 0.55$. The depth is consistent with a total eclipse, and the eclipse FWHM duration is $0.17 \pm 0.05$ of the orbital period. The folded light curve is quite flat; the peak-to-trough pulsed fraction, $P F_{\mathrm{pt}}=$ $\left(F_{\max }-F_{\min }\right) /\left(F_{\max }+F_{\min }\right)$, where $F_{\max }$ and $F_{\min }$ are the observed background-subtracted count rates at the peak and at the minimum, is $78 \pm 12 \%$ ( $1-\sigma$ error).

\subsection{X-ray position}

We used $20.4 \mathrm{ks}$ of PC mode data and simultaneous Swift/UVOT images to obtain an astrometrically-corrected position (see Evans et al. 2009; Goad et al. 2007) of: RA(J2000) = 250.46104, $\operatorname{Dec}(\mathrm{J} 2000)=-45.54092$, which is equivalent to: $\mathrm{RA}(\mathrm{J} 2000)=$ $16^{\mathrm{h}} 41^{\mathrm{m}} 50^{\mathrm{s}} 65, \operatorname{Dec}(\mathrm{J} 2000)=-45^{\circ} 32^{\prime} 27^{\prime \prime} .3$, with an uncertainty of $1^{\prime \prime} .9$ (90\% confidence level 2 . This position is $2^{\prime \prime} .5$ from the source 2MASS J16415078-4532253 and 4". 3 from the XMMNewton position (Walter et al.2006), and it allows us to confirm the 2MASS source as the optical counterpart of IGR J16418-4532.

\subsection{UVOT optical counterpart}

At the XRT position of IGR J16418-4532, no detection was achieved in any UV band down to a limit of $w 1>21.78$, $m 2>21.66, w 2>21.89 \mathrm{mag}$, respectively. A marginal detection $(2.9 \sigma)$ was obtained in the $u$ band, at $22.28 \pm 0.40$ mag. These magnitudes are on the UVOT photometric system described in Poole et al. (2008), and are not corrected for Galactic or intrinsic extinction. At this position, the column due to Galactic absorption is $1.59 \times 10^{22} \mathrm{~cm}^{-2}$ (Kalberla et al. 2005). By adopting the conversion into $A_{V}$ (Predehl \& Schmitt 1995), $N_{\mathrm{H}}=1.79 \times 10^{21} A_{V}$

2 This new UVOT-enhanced XRT position takes advantage of the new calibration of the XRT to UVOT detector co-ordinates of 2011 August Evans 2011); see, also: http://www.swift.ac.uk/reprocessed.php

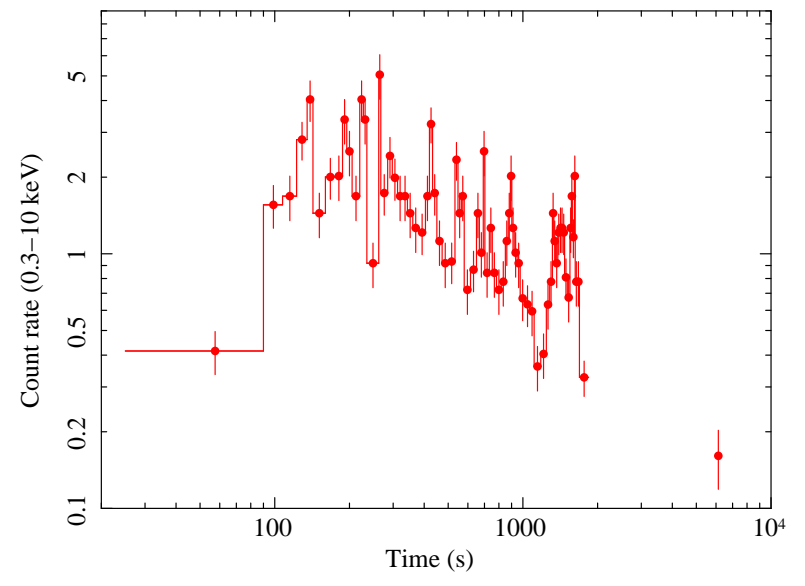

Figure 2. Swift/XRT $0.3-10 \mathrm{keV}$ light curve of IGR J16418-4532 during observation 00031929001, background-subtracted, corrected for pileup, PSF losses, and vignetting $(\mathrm{SNR}=5)$. Time is referred to the start of the observation.

$\mathrm{cm}^{-2}$, this corresponds to (Cardelli et al. 1989) $A_{u}=14.2 \mathrm{mag}$ in the UVOT $u$ band.

\subsection{Spin period search}

We looked for evidence of the $1246 \pm 100 \mathrm{~s}$ spin periodicity reported by Walter et al. (2006) in the Solar system barycentred XRT light curves. We only considered observations where the source was significantly detected (thus excluding obsIDs 00032037001 and 00032037004).

We searched each single observation longer than one spin period and the total combined dataset within $\pm 300 \mathrm{~s}$ (corresponding to a $\pm 3 \sigma$ error reported by Walter et al. 2006) by applying both a Fourier transform and a $Z_{n}^{2}$ analysis (a method that does not require the binning of the data; Buccheri et al. 1983). No statistically significant signal was detected either in the individual observations or in the combined dataset. With respect to the latter, the upper limit on the pulsed fraction, (defined as the semi-amplitude of the sinusoidal modulation divided by the mean count rate), computed according to Vaughan et al. (1994), is $42 \%$ at the $99 \%$ confidence level and for periods between 946 and $1546 \mathrm{~s}$.

This upper limit is only marginally compatible with the $(64 \pm$ 10) $\%$ value measured by Walter et al. (2006). We note however that they were able to detect pulsations only during a fraction of their XMM-Newton data and in particular while the source was not flaring, and that our data were collected in a variety of states of brightness and across almost all orbital phases. So our upper limit and their measure may not be straightforward to compare.

\subsection{Light curves}

Fig. 2] shows the $0.3-10 \mathrm{keV}$ light curve of IGR J16418-4532 during the 2001 February 18 observation, binned in order to achieve a SNR of 5 . The source reached $\sim 5$ counts $\mathrm{s}^{-1}$ during the initial flare and then decayed through a series of smaller flares. The lowest point is at 0.16 counts $\mathrm{s}^{-1}$. When binned at a SNR of 3 the dynamical range, during this observation, is of order 50 .

Fig. 3] shows the $0.3-10 \mathrm{keV}$ light curve of IGR J16418-4532 of the whole 2011 July campaign after bin-by-bin backgroundsubtraction and corrections for pile-up, PSF losses, and vignetting, 


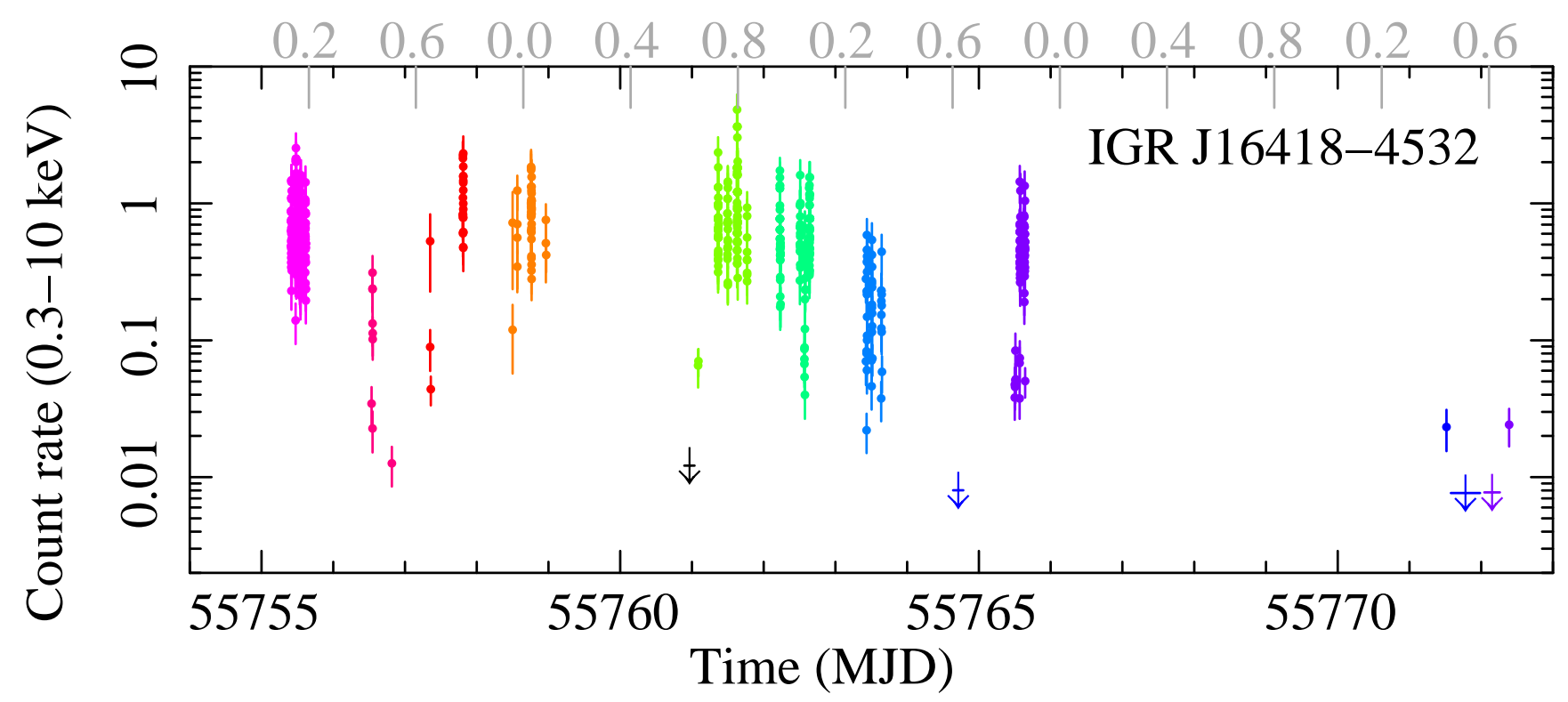

Figure 3. Swift/XRT 0.3-10 keV light curve of IGR J16418-4532 during the 2011 July monitoring program, background-subtracted and corrected for pile-up, $\mathrm{PSF}$ losses, and vignetting $(\mathrm{SNR}=3$ ). Downward-pointing arrows are $3 \sigma$ upper limits. Different colours (see the electronic edition) mark different observations (see Table 1). The top axis reports the phase (with $P_{\text {orb }}=3.73886 \mathrm{~d}$ and $T_{\text {epoch }}=$ MJD 53560.20000).

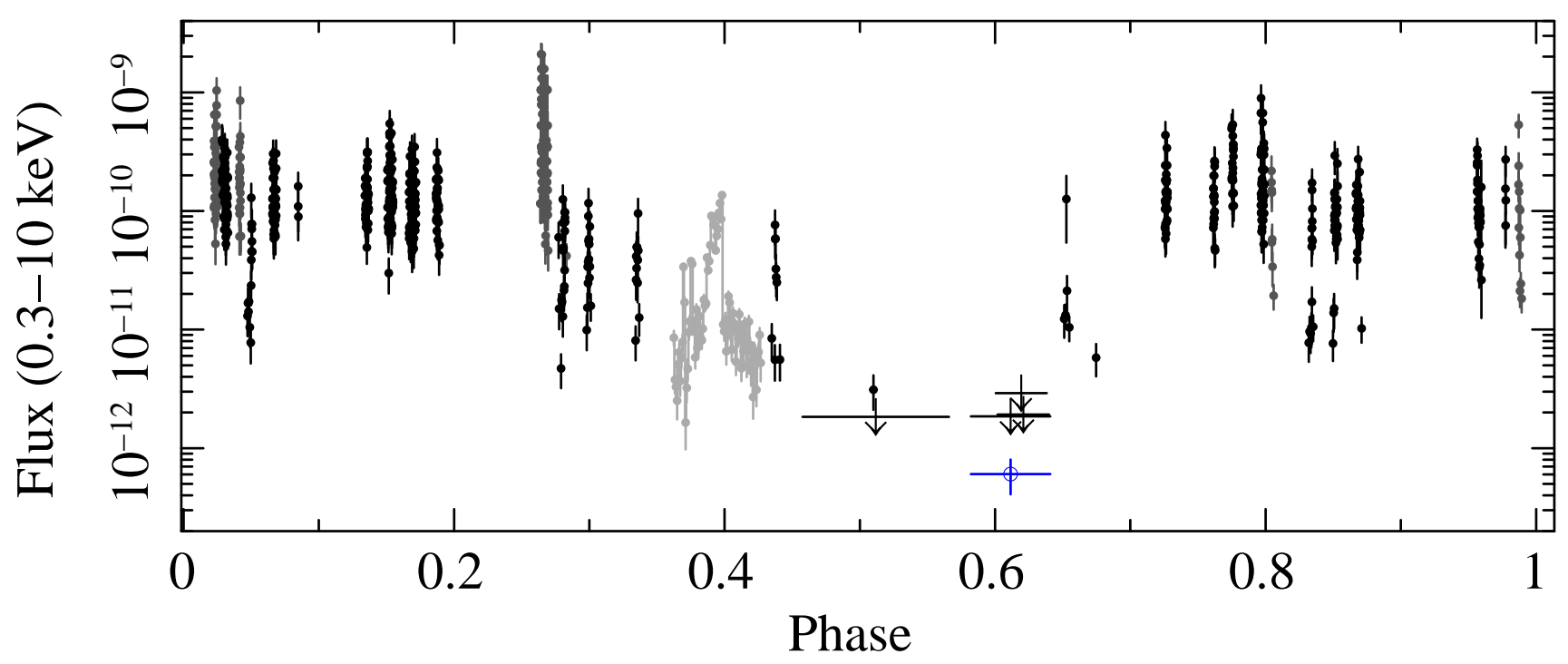

Figure 4. Swift/XRT $0.3-10 \mathrm{keV}$ flux light curve of IGR J16418-4532, folded at $P_{\text {orb }}=3.73886 \mathrm{~d}$ and $T_{\text {epoch }}=$ MJD 53560.20000. The data were collected in 2011 February and June (dark grey filled circles), and 2011 July (black filled circles). Downward-pointing arrows are $3 \sigma$ upper limits. The open blue circle is a detection obtained by combining three observations (00032037001, 00032037004, and snapshots 1-3 of 00032037007). Also included are the 2004 August XMM data (light grey curve).

binned at $\mathrm{SNR}=3$. The light curve starts at phase 0.16 (assuming a period of $P_{\text {orb }}=3.73886 \mathrm{~d}$ and an initial epoch $T_{\text {epoch }}=$ MJD 53560.20000) and monitors the source state through over three full periods. Superimposed on the long-term orbital modulation, which follows that seen in the BAT data, flaring is observed on short time scales, as shown in Fig. 2 This behaviour has been observed in most SFXTs (Sidoli et al. 2008; Romano et al. 2009b, 2011a, 2010).

The lowest point (as observed by XRT) was collected during the eclipse, by combining three observations (00032037001,
00032037004, and snapshot $31-3$ of 00032037007, see open blue point in Fig. (4) at $2.5 \times 10^{-3}$ counts $\mathrm{s}^{-1}$. This corresponds to an unabsorbed $0.3-10 \mathrm{keV}$ flux of $\sim 6.0 \times 10^{-13} \mathrm{erg} \mathrm{cm}^{-2} \mathrm{~s}^{-1}$, and to a luminosity of $1.2 \times 10^{34} \mathrm{erg} \mathrm{s}^{-1}$ (assuming the optical counterpart distance of $13 \mathrm{kpc}$ ). The lowest point during the campaign outside the eclipse was recorded on MJD 55763.43 at 0.022 counts $\mathrm{s}^{-1}$,

\footnotetext{
3 Part of an observation with a continuous viewing of the target, with typical timescales of $10^{3} \mathrm{~s}$.
} 
IGR J16418-4532 with Swift
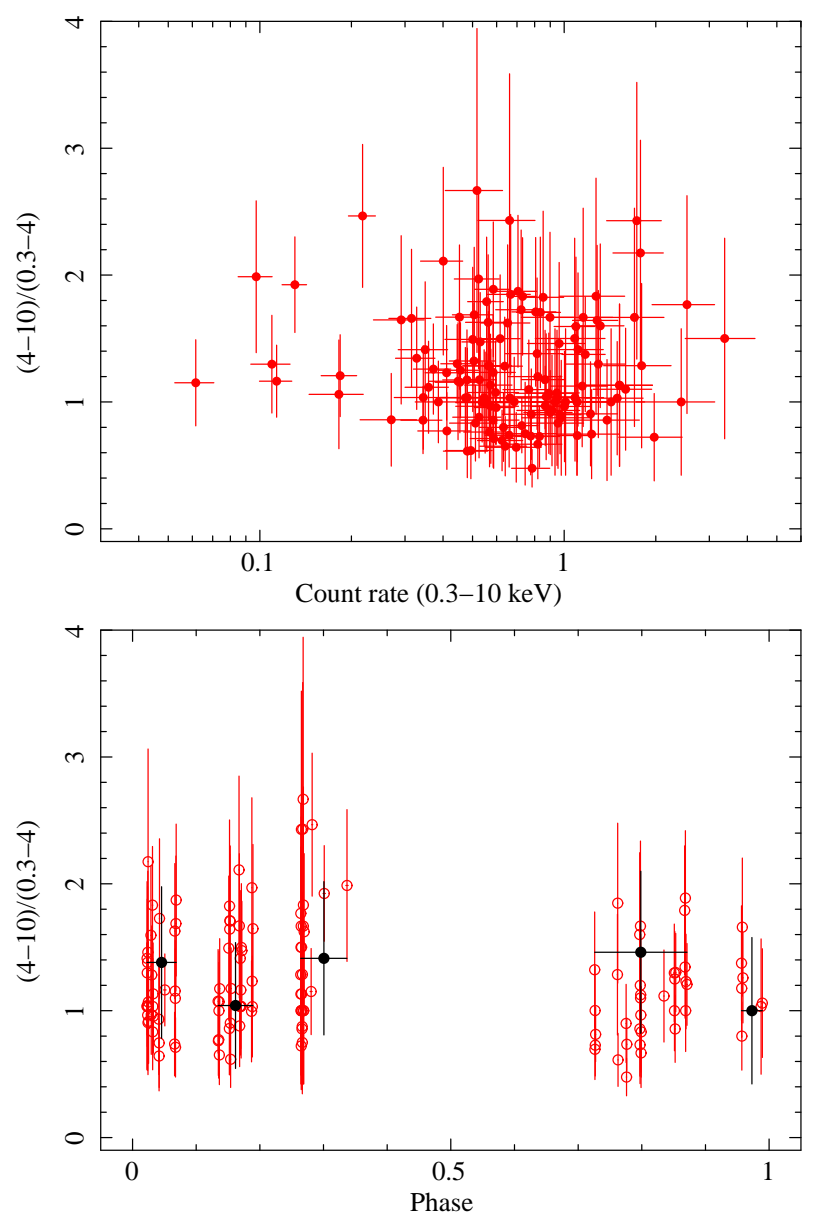

Figure 5. Swift/XRT hardness ratio (red open circles, 30 counts per bin) as a function of count rate (top) and phase (bottom). The black points are weighted means.

corresponding to an unabsorbed $0.3-10 \mathrm{keV}$ flux of $\sim 4.7 \times 10^{-12}$ erg $\mathrm{cm}^{-2} \mathrm{~s}^{-1}$, and to a luminosity of $9.5 \times 10^{34} \mathrm{erg} \mathrm{s}^{-1}$. As the peak count rate is reached on MJD 55610.08 at $\gtrsim 8$ counts $\mathrm{s}^{-1}$ the observed dynamical range of this source is at least 370, (1400 considering the points within the eclipse).

Fig. 4 shows the $0.3-10 \mathrm{keV}$ light curve, folded using $P_{\text {orb }}=$ $3.73886 \mathrm{~d}$ and $T_{\text {epoch }}=$ MJD 53560.20000. We adopted count rate to flux conversion factors derived from spectral fits of each observation (Section 3.6) and subsequent interpolation across observations where fitting was not feasible. We also include the $\sim 10 \mathrm{ks}$ 2004 August 19 XMM-Newton/EPIC-pn observation (Walter et al. 2006), binned at $256 \mathrm{~s}$, and converted into flux by adopting a single conversion factor obtained from fitting the mean spectrum with an absorbed power-law model $\left(\Gamma=1.40 \pm 0.05, N_{\mathrm{H}}=\right.$ $(10.5 \pm 0.5) \times 10^{22} \mathrm{~cm}^{-2}, 1-\sigma$ errors; $\chi^{2}=0.99 / 169$ dof $)$. The eclipse lasts $\Delta \phi \sim 0.2$ and is centred at $\phi \sim 0.55$, as observed in the BAT data (Fig. 1).

Fig. 5 shows the hardness ratio (HR defined as $\mathrm{H} / \mathrm{S}$, where $\mathrm{H}$ is the count rate in the $4-10 \mathrm{keV}$ band and $\mathrm{S}$ is the count rate in the $0.3-4 \mathrm{keV}$ band), as a function of count rate (top), and orbital phase (bottom), where the light curve was binned to have 30 counts per bin (red open circles). A fit to a constant value yields a hardness ratio value of $1.01 \pm 0.03$ and $\chi_{\nu}^{2}=0.85$ for 130 degrees of freedom (dof), so that the HR is shown to be roughly constant over two orders of magnitude in count rate. Furthermore, the HR were also
Table 2. XRT spectroscopy of IGR J16418-4532.

\begin{tabular}{lcccrr}
\hline ObsID & $\begin{array}{c}N_{\mathrm{H}} \\
\left(10^{22} \mathrm{~cm}^{-2}\right)\end{array}$ & $\Gamma$ & Flux $^{a}$ & $\mathrm{~L}^{b}$ & $\begin{array}{r}\chi_{\nu}^{2} / \mathrm{dof} \\
\text { C-stat }(\mathrm{dof})^{c}\end{array}$ \\
\hline 00031929001 & $6.77_{-1.78}^{+2.32}$ & $1.38_{-0.54}^{+0.61}$ & $21.36_{-2.44}^{+3.14}$ & 43 & $0.532 / 18$ \\
00031929002 & $4.17_{-1.24}^{+1.70}$ & $1.04_{-0.45}^{+0.51}$ & $11.58_{-1.30}^{+1.39}$ & 23 & $0.845 / 17$ \\
00043105001 & $5.28_{-2.54}^{+3.47}$ & $1.35_{-0.87}^{+0.99}$ & $3.85_{-0.83}^{+1.16}$ & 8 & $70.05(76)$ \\
00031929003 & $5.20_{-0.82}^{+1.00}$ & $1.35_{-0.24}^{+0.27}$ & $8.27_{-0.58}^{+0.65}$ & 17 & $0.935 / 58$ \\
00031929004 & $5.16_{-2.81}^{+4.17}$ & $0.09_{-0.75}^{+0.87}$ & $1.05_{-0.17}^{+0.21}$ & 2 & $169.46(141)$ \\
00031929007 & $3.93_{-0.97}^{+1.24}$ & $1.04_{-0.36}^{+0.40}$ & $7.62_{-0.69}^{+0.72}$ & 15 & $0.647 / 28$ \\
00032037002 & $4.59_{-1.00}^{+1.23}$ & $1.05_{-0.34}^{+0.38}$ & $6.32_{-0.54}^{+0.56}$ & 13 & $0.831 / 31$ \\
00032037003 & $5.65_{-1.96}^{+2.80}$ & $0.62_{-0.55}^{+0.62}$ & $2.51_{-0.31}^{+0.32}$ & 5 & $1.114 / 16$ \\
00032037005 & $5.14_{-1.39}^{+1.72}$ & $1.01_{-0.40}^{+0.45}$ & $3.88_{-0.37}^{+0.40}$ & 8 & $0.838 / 27$ \\
\hline & & & & &
\end{tabular}

${ }^{\text {a }}$ Unabsorbed 2-10 keV fluxes $\left(10^{-11} \mathrm{erg} \mathrm{cm}^{-2} \mathrm{~s}^{-1}\right)$.

b $2-10 \mathrm{keV}$ luminosities in units of $10^{35} \mathrm{erg} \mathrm{s}^{-1}$, at $13 \mathrm{kpc}$.

${ }^{\mathrm{c}}$ Cash statistics (C-stat).

weighted across several phase intervals (black points); we cannot find evidence of variations of the HR as a function of phase.

\subsection{Spectral analysis}

We first extracted PC spectra for each segment in which a detection was obtained and a minimum of $\sim 100$ source counts were available. We did not consider the WT data for our spectroscopic study as they were statistically poor and were heavily contaminated by the background from the single-reflection rings from GX 340.0+0. The data were rebinned with a minimum of 20 counts per energy bin to allow $\chi^{2}$ fitting, with the exception of low-count-statistics spectra, in which Cash (Cash 1979) statistics and spectra binned to 1 count per bin were used, instead. The spectra were fit in the $0.3-10 \mathrm{keV}$ energy range with a single absorbed power law model. More complex models were not required by the data. The results are reported in Table 2. We note that the spectral parameters do not show significant variability within the large uncertainties, despite the large observed variations in flux throughout the campaign.

Observation 00031929001 (shown as the dark grey filled circles at phase 0.27 in Fig. 4 and detailed in Fig. 2), in particular, deserves special attention. Although it was performed as a followup of a false trigger (later attributed to the nearby GX $340+0$ ), it nonetheless found the source in a very active state, the brightest of the whole campaign, with its average $2-10 \mathrm{keV}$ unabsorbed flux of $\sim 2 \times 10^{-10} \mathrm{erg} \mathrm{cm}^{-2} \mathrm{~s}^{-1}$, corresponding to $\sim 4 \times 10^{36} \mathrm{erg} \mathrm{s}^{-1}$ at $13 \mathrm{kpc})$. The peak flux was $\sim 2 \times 10^{-9} \mathrm{erg} \mathrm{cm}^{-2} \mathrm{~s}^{-1}\left(\sim 4 \times 10^{37}\right.$ $\left.\mathrm{erg} \mathrm{s}^{-1}\right)$. An absorbed power-law model yielded a high absorbing column $N_{\mathrm{H}}=\left(6.8_{-1.8}^{+2.3}\right) \times 10^{22} \mathrm{~cm}^{-2}$ (in excess of the Galactic one, $1.59 \times 10^{22} \mathrm{~cm}^{-2}$ ) and a photon index $\Gamma=1.4_{-0.5}^{+0.6}$ (see Table 2).

\section{DISCUSSION}

IGR J16418-4532 is a candidate SFXT, based on the hard X-ray behaviour (Sguera et al. 2006), for which we know both the orbital $\left(P_{\text {orbit }} \sim 3.7 \mathrm{~d}\right.$, Corbet et al. 2006) and the spin $(\sim 1250 \mathrm{~s}$, Walter et al. 2006) periods. These values place IGR J16418-4532 in the region of the Corbet diagram (Corbet 1986) of the windaccreting supergiant high-mass X-ray binaries. In this Paper we 
have taken advantage of the short orbital period which makes IGR J16418-4532 a very good source to monitor with the XRT, to study the X-ray properties of this source as a function of the orbital phase, in particular, to address the issue of its SFXT nature.

The Swift/BAT and XRT folded light curves of IGR J16418-4532 (Fig. 11 and Fig. 4) show the presence of a dip that is consistent with zero intensity. When interpreted as an eclipse, it can be used to infer the nature of the primary of the binary system. The BAT lightcurve of IGR J16418-4532 (excluding the eclipse) does not clearly show a flux modulation with the orbital phase, suggesting that the eccentricity is not large. This is in agreement with the observation that in many X-ray binary systems with nearly circular orbits and short orbital periods ( $\leqslant 4$ days), the eccentric orbit produced by the explosion of the supernova is circularized on timescale of $10^{6} \mathrm{yr}$ (consistent with the evolution timescale of an $\mathrm{O}$ star), due to viscous tidal interactions (see e.g. Press et al. 1975). Thus, for simplicity, we assume a circular orbit $\left(P_{\text {orb }}=3.73886 \mathrm{~d}\right)$. Let us adopt the typical values of stellar masses and radii (Martins et al. 2005) for a primary of spectral type O8.5 and luminosity classes I, III, or $\mathrm{V}$, and for the neutron star a mass of $M_{\mathrm{NS}}=1.4 \mathrm{M}_{\odot}$, and a radius of $R_{\mathrm{NS}}=10 \mathrm{~km}$. By combining Kepler's third law with the relationship given by Rappaport \& Joss (1983) for the radius of the primary $R$,

$(R / a)^{2}=\cos ^{2} i+\sin ^{2} i \sin ^{2} \theta_{\mathrm{e}}$

where $a$ is the semi-major axis of the orbit, $i$ is the inclination, and $\theta_{\mathrm{e}}$ is the eclipse semi-angle, we find that the observed $\Delta \phi=$ 0.2 is inconsistent with a luminosity class III or V primary. We therefore conclude that indeed the primary is an O8.5I supergiant star (with mass, radius, luminosity, effective temperature given by Martins et al. (2005), $M_{\mathrm{OB}}=31.5 \mathrm{M}_{\odot}, R_{\mathrm{OB}}=21.4 \mathrm{R}_{\odot}$, $\left.\log L_{\mathrm{OB}} / L_{\odot}=5.65, T_{\text {eff }}=32274 \mathrm{~K}\right)$. Therefore, the distance to IGR J16418-4532 is $\sim 13 \mathrm{kpc}$ (Rahoui et al. 2008).

During the eclipse of IGR J16418-4532, we detect a non-zero flux with the XRT ( $L_{\mathrm{X}} \sim 3 \times 10^{34} \mathrm{erg} \mathrm{s}^{-1}$, see Fig. 4). Detections of HMXBs during the eclipse are quite common (see e.g. van der Meer et al. 2005; Clark et al. 1988; Sako et al. 1999) and can be ascribed to X-ray photons scattered by the stellar wind of the $\mathrm{OB}$ star and discrete fluorescent and recombination lines from highly ionized gas around the compact object. The decrease in $\mathrm{X}$ ray flux, when the compact object is approaching the eclipse, is related to the increase of the column density along the line of sight, through the stellar wind produced by the OB star. From observations of other wind-fed X-ray pulsars, such as 4U 1700-377, Vela X-1, OAO 1657-415 (see e.g. van der Meer et al. 2005; Sako et al. 1999; Audley et al. 2006) the absorption column density during the eclipse ranges from $\approx 10^{23} \mathrm{~cm}^{-2}$ up to $\approx 10^{24} \mathrm{~cm}^{-2}$.

We can compare the X-ray luminosity and absorption column density of IGR J16418-4532 during the eclipse with those calculated by van der Meer et al. (2005) for 4U 1700-377, a windfed HMXBs with properties similar to IGR J16418-4532 (orbital period $P_{\text {orb }}=3.4 \mathrm{~d}$, companion star of spectral type O6.5Iaf). van der Meer et al. (2005) observed 4U 1700-377 with XMMNewton during an eclipse; they found an X-ray luminosity of $\approx 7 \times 10^{34} \mathrm{erg} \mathrm{s}^{-1}$ (in agreement with the X-ray luminosity of IGR J16418-4532 during the eclipse) and a column density of $\approx 10^{24} \mathrm{~cm}^{-2}$.

The XRT light curve (Figs. 2 and 3 ) shows a large dynamic range, up to 1-2 orders of magnitude within a single snapshot and about 3 orders of magnitude overall (even when the low-intensity state is excluded). While this dynamic range is lower than that ob-

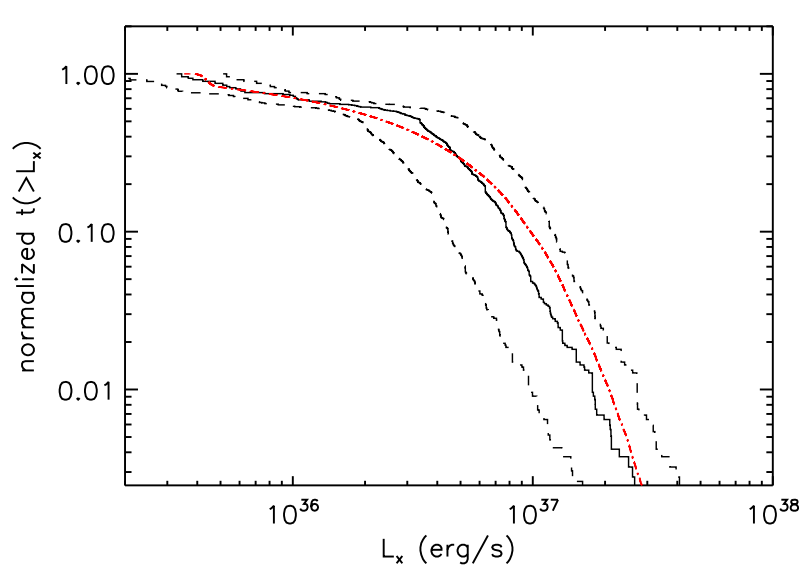

Figure 6. Observed cumulative luminosity distribution (solid black line) and relative uncertainties (dashed black lines), compared with a possible solution (red dot-dashed red line; see text).

Table 3. Wind parameters values for IGR J16418-4532.

\begin{tabular}{lcc}
\hline Parameter & min. value & max. value \\
\hline$\dot{M}_{\text {tot }}$ & $4 \times 10^{-7} \mathrm{M}_{\odot} \mathrm{yr}^{-1}$ & $8 \times 10^{-7} \mathrm{M}_{\odot} \mathrm{yr}^{-1}$ \\
$f$ & 0.85 & 0.95 \\
$v_{\infty}$ & $800 \mathrm{~km} \mathrm{~s}^{-1}$ & $1300 \mathrm{~km} \mathrm{~s}^{-1}$ \\
$\beta$ & 0.8 & 1.2 \\
$\zeta$ & 0.8 & 1.6 \\
$\gamma$ & -1.5 & -0.5 \\
$M_{\mathrm{a}}$ & $5 \times 10^{16} \mathrm{~g}$ & $5 \times 10^{19} \mathrm{~g}$ \\
$M_{\mathrm{b}}$ & $5 \times 10^{19} \mathrm{~g}$ & $10^{21} \mathrm{~g}$ \\
\hline
\end{tabular}

served in the classical (Chaty et al. 2010) SFXTs, which reach 4-5 orders of magnitude in dynamic range (e.g. IGR J17544-2619), it is indeed typical of intermediate SFXTs (e.g., IGR J18483-0311). The 2011 February flare (Fig. 2 and dark grey filled circles at phase 0.27 in Fig. 4), in particular, reached $\sim 2 \times 10^{-9} \mathrm{erg}$ $\mathrm{cm}^{-2} \mathrm{~s}^{-1}$ (2-10 keV, unabsorbed), corresponding to a luminosity of $\sim 4 \times 10^{37} \mathrm{erg} \mathrm{s}^{-1}$, which is typical of SFXT outbursts as we have observed them with Swift/XRT (e.g., Romano et al. 2008, 2009a; Sidoli et al. 2009b a).

On the other hand, while the typical SFXT light curve is characterized by a faint flaring activity with luminosity of $10^{33}-$ $10^{34} \mathrm{erg} \mathrm{s}^{-1}$ (Sidoli et al. 2008; Romano et al. 2009b, 2011a), sporadically interrupted by flares with duration of a few hours and peak luminosity of $10^{36}-10^{37} \mathrm{erg} \mathrm{s}^{-1}$, IGR J16418-4532 exhibits a flaring activity ranging from $\sim 10^{35} \mathrm{erg} \mathrm{s}^{-1}$ up to $\sim$ $4 \times 10^{37} \mathrm{erg} \mathrm{s}^{-1}$ (also see Walter et al.2006 and the light grey curve in Fig. (4).

To reproduce the observed X-ray variability, we applied the clumpy wind model of Ducci et al. (2009), which we briefly summarize here. This model assumes that the wind of OB supergiants is inhomogeneous, composed of dense clumps surrounded by a hotter and homogeneous wind. Clumps follow a power-law mass distribution

$p\left(M_{\mathrm{cl}}\right)=k\left(\frac{M_{\mathrm{cl}}}{M_{\mathrm{a}}}\right)^{-\zeta}$,

where $k=(1-\zeta) M_{\mathrm{a}}^{-\zeta} /\left(M_{\mathrm{b}}^{1-\zeta}-M_{\mathrm{a}}^{1-\zeta}\right)$ is the normalization constant, $M_{\mathrm{cl}}$ is the mass of a clump, and $M_{\mathrm{a}}$ and $M_{\mathrm{b}}$ de- 
fine the extrema of the mass range. Assuming a spherical geometry for the clumps, the initial clump dimension distribution is given by $\dot{N}_{M_{c l}} \propto R_{\mathrm{cl}}^{\gamma}$ clumps s$^{-1}$, where $R_{\mathrm{cl}}$ is the radius of the clump. The clump mass loss rate $\dot{M}_{\mathrm{cl}}$ is related to the total mass loss rate $\dot{M}_{\text {tot }}$ by means of $f=\dot{M}_{\mathrm{cl}} / \dot{M}_{\mathrm{tot}}$, the fraction of wind mass in the form of clumps. Clumps and the homogeneous wind follow the $\beta$ velocity law $v(r)=v_{\infty}\left(1-0.9983 \frac{R_{\mathrm{OB}}}{r}\right)^{\beta}$ (Castor et al. 1975), where $\beta$ is a constant in the range $\sim 0.5-1.5, R_{\mathrm{OB}}$ is the radius of the supergiant and $v_{\infty}$ is the terminal wind velocity.

IGR J16418-4532 was intensely monitored by Swift/XRT for two orbital cycles (MJD 55755-55766). Each snapshot has a duration of $\sim 1 \mathrm{ks}$, for a total of up to $\sim 5 \mathrm{ks}$ per day, which is the typical timescale for the flare durations observed in SFXTs (Sguera et al. 2006). For this reason, we cannot establish the number of observed flares and their duration, therefore we cannot use this information to compare the observed lightcurve with that calculated with the clumpy wind model of Ducci et al. (2009), as has been done in previous work (Ducci et al.2009; Romano et al.2010; Ducci et al. 2010). However, it is possible to compare the observed cumulative luminosity distribution out of eclipse with that calculated with the clumpy wind model. For the former, the Swift/XRT count rate, measured in the $0.3-10 \mathrm{keV}$, was converted to the $0.1-$ $100 \mathrm{keV}$ luminosity using the average spectral parameters obtained by Walter et al. (2006) and Ducci et al. (2010), and assuming a distance of $13 \mathrm{kpc}$. For the latter, we adopted the physical parameters for the O8.5I primary and the neutron star secondary reported above, and the force multiplier parameters (see Ducci et al. 2009) obtained by Shimada et al. (1994) for a O8.5I star $(k=0.375$, $\alpha=0.522, \delta=0.099$ ).

Fig. 6 shows the Swift/XRT cumulative luminosity distribution (black solid line) with its $90 \%$ uncertainties (black dashed lines). Fig. 6 also shows the cumulative luminosity distribution (red dot-dashed line) calculated with the clumpy wind model by adopting the following parameter values: mass loss rate $\dot{M}_{\text {tot }}=$ $6 \times 10^{-7} \mathrm{M}_{\odot} \mathrm{yr}^{-1}$, terminal velocity $v_{\infty}=1000 \mathrm{~km} \mathrm{~s}^{-1}, \beta=1$, fraction of mass lost in clumps $f=0.9$, mass distribution powerlaw index $\zeta=1.1$, power-law index of the initial clump dimension distribution $\gamma=-1$, minimum clump mass $M_{\mathrm{a}}=5 \times 10^{18} \mathrm{~g}$ and maximum clump mass $M_{\mathrm{b}}=10^{20} \mathrm{~g}$. Further acceptable solutions can be found assuming the wind parameters in the ranges reported in Table 3

The wind parameters obtained for IGR J16418-4532 are roughly in agreement with those of HMXBs previously studied (see Ducci et al. 2009; Romano et al. 2010; Ducci et al. 2010), with the exception of $f$ and $v_{\infty}$. The obtained low value of the terminal velocity can be explained as follows: when the wind is highly ionized by the X-ray photons emitted by the neutron star, the high ionization modifies the dynamics of the line-driven stellar wind of $\mathrm{OB}$ supergiants (especially in close binary systems), leading to a reduction of the wind velocity in the direction of the neutron star (see e.g. Stevens \& Kallman 1990 and references therein). The mass loss rate is lower than the typical mass loss rate of O8.5I stars, which is of the order of $\approx 2 \times 10^{-6} \mathrm{M}_{\odot} \mathrm{yr}^{-1}$ (Vink et al. 2000), but in agreement with the hypothesis that the mass loss rates derived from homogeneous-wind model measurements with the $\mathrm{H} \alpha$ method are overestimated by a factor of 2-10 if the wind is clumpy (see e.g. Lépine \& Moffat 2008). However, we cannot exclude the possibility that the X-ray variability of IGR J16418-4532 is not totally due to the accretion of an inhomogeneous wind. In fact, other mechanisms could be at work, like e.g. transient accretion discs (Taam et al. 1988) and intermittent accretion flow onto a neutron star (Lamb et al. 1977; Ducci et al. 2010).
Our results, based on our intense monitoring of IGR J16418-4532, show that its X-ray properties (dynamical range, flaring activity and peak luminosity) are consistent with an intermediate SFXT nature.

\section{ACKNOWLEDGMENTS}

We thank the Swift team duty scientists and science planners. We also thank the remainder of the Swift XRT and BAT teams, S. Barthelmy and J.A. Nousek, in particular, for their invaluable help and support of the SFXT project as a whole. We thank A. Cucchiara for helpful discussions. We acknowledge financial contribution from the agreement ASI-INAF I/009/10/0. This work was supported at PSU by NASA contract NAS5-00136. PE acknowledges financial support from the Autonomous Region of Sardinia through a research grant under the program PO Sardegna FSE 2007-2013, L.R. 7/2007 "Promoting scientific research and innovation technology in Sardinia". We also thank the anonymous referee for swift comments that helped improve the paper.

\section{REFERENCES}

Audley M. D., Nagase F., Mitsuda K., Angelini L., Kelley R. L., 2006, MNRAS, 367, 1147

Barthelmy S. D., Barbier L. M., Cummings J. R. et al., 2005, Space Science Reviews, 120, 143

Bozzo E., Stella L., Ferrigno C., Giunta A., Falanga M., Campana S., Israel G., Leyder J. C., 2010, A\&A, 519, A6

Buccheri, R. et al., 1983, A\&A, 128, 245

Burrows D. N., Hill J. E., Nousek J. A. et al., 2005, Space Science Reviews, 120, 165

Cardelli J. A., Clayton G. C., Mathis J. S., 1989, ApJ, 345, 245

Cash W., 1979, ApJ, 228, 939

Castor J. I., Abbott D. C., Klein R. I., 1975, ApJ, 195, 157

Chaty S., Rahoui F., Foellmi C., Tomsick J. A., Rodriguez J., Walter R., 2008, A\&A, 484, 783

Chaty S., Zurita Heras J. A., Bodaghee A., 2010, ArXiv e-prints, 1012.2318

Clark G. W., Minato J. R., Mi G., 1988, ApJ, 324, 974

Corbet R. et al., 2006, The Astronomer's Telegram, 779, 1

Corbet R. H. D., 1986, MNRAS, 220, 1047

Ducci L., Sidoli L., Mereghetti S., Paizis A., Romano P., 2009, MNRAS, 398, 2152

Ducci L., Sidoli L., Paizis A., 2010, MNRAS, 408, 1540

Evans P. A., 2011, GRB Coordinates Network, Circular Service, 12150,

Evans P. A. et al., 2009, MNRAS, 397, 1177

Goad M. R. et al., 2007, A\&A, 476, 1401

in't Zand J. J. M., 2005, A\&A, 441, L1

Kalberla P. M. W., Burton W. B., Hartmann D., Arnal E. M., Bajaja E., Morras R., Pöppel W. G. L., 2005, A\&A, 440, 775

Krimm H., Barbier L., Barthelmy S. D., et al. 2006, Astron. Tel., 904

Krimm H. A., Barthelmy S. D., Cummings J. R., Markwardt C. B., Skinner G., Tueller J., Swift/BAT Team 2008, in AAS/High Energy Astrophysics Division Vol. 10 of AAS/High Energy Astrophysics Division, Status of the Swift/BAT Hard X-ray Transient Monitor. p. \#07.01

Lamb F. K., Fabian A. C., Pringle J. E., Lamb D. Q., 1977, ApJ, 217,197 
Lépine S., Moffat A. F. J., 2008, AJ, 136, 548

Levine A. M., Bradt H. V., Chakrabarty D., Corbet R. H. D., Harris R. J., 2010, ArXiv e-prints, 1009.0450

Martins F., Schaerer D., Hillier D. J., 2005, A\&A, 436, 1049

Negueruela I., Smith D. M., Reig P., Chaty S., Torrejón J. M., 2006, 604, 165

Poole T. S., Breeveld A. A., Page et al. M. J., 2008, MNRAS, 383, 627

Predehl P., Schmitt J. H. M. M., 1995, A\&A, 293, 889

Press W. H., Smarr L. L., Wiita P. J., 1975, ApJL, 202, L135

Rahoui F., Chaty S., Lagage P.-O., Pantin E., 2008, A\&A, 484, 801

Rappaport S. A., Joss P. C., 1983, in Lewin W. H. G., van den Heuvel E. P. J., eds, Accretion-Driven Stellar X-ray Sources Xray pulsars in massive binary systems, 1

Romano P., La Parola V., Vercellone S. et al., 2011a, MNRAS, 410, 1825

Romano P., Mangano V., Esposito P. et al., 2011b, Astron. Tel., 3174, 1

Romano P., Sidoli L., Cusumano G. et al., 2009a, MNRAS, 392, 45

Romano P., Sidoli L., Cusumano G. et al., 2009b, MNRAS, 399, 2021

Romano P., Sidoli L., Ducci L. et al., 2010, MNRAS, 401, 1564

Romano P., Sidoli L., Mangano V. et al., 2008, ApJL, 680, L137

Roming P. W. A., Kennedy T. E., Mason et al. K. O. et al., 2005, Space Science Reviews, 120, 95

Sako M., Liedahl D. A., Kahn S. M., Paerels F., 1999, ApJ, 525, 921

Sguera V., Barlow E. J., Bird A. J. et al., 2005, A\&A, 444, 221

Sguera V., Bazzano A., Bird A. J. et al., 2006, ApJ, 646, 452

Shimada M. R., Ito M., Hirata B., Horaguchi T., 1994, in Balona L. A., Henrichs H. F., Le Contel J. M., eds, Pulsation; Rotation; and Mass Loss in Early-Type Stars Vol. 162 of IAU Symposium, Radiatively driven winds of OB stars, 487

Sidoli L., Romano P., Esposito P. et al., 2009a, MNRAS, 400, 258

Sidoli L., Romano P., Mangano V. et al., 2009b, ApJ, 690, 120

Sidoli L., Romano P., Mangano V. et al., 2008, ApJ, 687, 1230

Stevens I. R., Kallman T. R., 1990, ApJ, 365, 321

Taam R. E., Brown D. A., Fryxell B. A., 1988, ApJL, 331, L117

Tomsick J. A., Lingenfelter R., Corbel S., Goldwurm A., Kaaret P., 2004, Astron. Tel., 224

van der Meer A., Kaper L., di Salvo T., Méndez M., van der Klis M., Barr P., Trams N. R., 2005, A\&A, 432, 999

Vaughan B. A., van der Klis M., Wood K. S. et al., 1994, ApJ, 435,362

Vink J. S., de Koter A., Lamers H. J. G. L. M., 2000, A\&A, 362, 295

Walter R., Zurita Heras J., Bassani L. et al., 2006, A\&A, 453, 133

This paper has been typeset from a $\mathrm{T}_{\mathrm{E}} \mathrm{X} / \mathrm{LT}_{\mathrm{E}} \mathrm{X}$ file prepared by the author. 